\title{
Are Profiles of Adaptive and Maladaptive Anger Regulation Differently Related to Adjustment in Early Adolescence?
}

\author{
Nantje Otterpohl'1, Malte Schwinger ${ }^{2}$, Elke Wild ${ }^{1}$ \\ ${ }^{1}$ Department of Psychology and Sport, Bielefeld University, Bielefeld, Germany \\ ${ }^{2}$ Department of Psychology, Witten/Herdecke University, Witten, Germany \\ Email: nantje.otterpohl@uni-bielefeld.de
}

Received 28 April 2015; accepted 9 June 2015; published 12 June 2015

Copyright (C) 2015 by authors and Scientific Research Publishing Inc.

This work is licensed under the Creative Commons Attribution International License (CC BY). http://creativecommons.org/licenses/by/4.0/

(c) (i) Open Access

\begin{abstract}
Adaptive and maladaptive emotion regulation (ER) strategies have been found to be important prerequisites of children's social competence and psychosocial adjustment. However, only a few studies have been conducted to examine their interactional impact on children's psychosocial adjustment. We collected multiple informant questionnaire data from $N=608$ adolescents $(10$ - 14 years) and their parents in order to examine the prevalence of adolescents' anger regulation profiles by means of latent profile analysis (LPA) as a sophisticated person-centered method. Overall, LPA identified four corresponding anger regulation profiles for adolescents (self-report) and parents (other-report). Furthermore, the different anger regulation profiles were found to be divergently related to internalizing and externalizing problems and prosocial behavior. Our findings support the assumption that specific kinds of psychopathology might be characterized by a different interplay of adaptive and maladaptive ER strategies. Possible reasons and practical implications are discussed.
\end{abstract}

\section{Keywords}

Emotion Regulation, Latent Profile Analysis, Internalizing, Externalizing, Prosocial Behavior

\section{Introduction}

The experience of emotions represents an evolutionary tool that helps us to understand the world in general. An important life task refers to regulating one's emotions in order to accommodate social conventions and affordances. There has been increased recognition of emotion regulation (ER) processes, with a special focus on how 
children can learn to manage their emotions in a socially appropriate manner (Morris, Silk, Steinberg, Myers, \& Robinson, 2007). Whereas children's and adolescents' use of several adaptive and maladaptive ER strategies (e.g., acceptance, rumination) has been related to their psychological development and adjustment (Adrian, Zeman, \& Veits, 2011; Eisenberg, Spinrad, \& Eggum, 2010; Southam-Gerow \& Kendall, 2002), less research has been conducted on the interplay between adaptive and maladaptive ER strategies (for an exception, see Aldao \& Nolen-Hoeksema, 2012). Little is known about how children and adolescents organize these different types of strategies within their own individual ER profile. Likewise, psychosocial correlates of such profiles have not yet been examined. In the present paper, we aim to contribute to the growing field of ER research by analyzing the prevalence and functionality of ER profiles in a multiple reporter study with a large sample of 6th-grade students. Given recommendations to examine discrete emotions rather than to examine general positive or negative affect (Zeman, Klimes-Dougan, Cassano, \& Adrian, 2007), we focus on the regulation of anger as a specific emotion in this article.

\subsection{Research on Emotion Regulation}

A widely accepted definition of ER has been suggested by Thompson (1994, p. 27): "Emotion regulation consists of internal and external processes involved in initiating, maintaining, and modulating the occurrence, intensity, and expression of emotions, especially their intensive and temporal features, to accomplish one’s goals.” A recent meta-analysis (Aldao, Nolen-Hoeksema, \& Schweizer, 2010) revealed that six ER strategies have been most commonly considered in ER research. Reappraisal comprises generating positive interpretations of a stressful situation in order to reduce distress. Problem-solving is often defined as an orientation or specific action directed at solving a problem (e.g., brainstorming) in order to modify or eliminate stressors. Both reappraisal and problem-solving have been found to be associated with higher scores in self-reported well-being and to be important predictors of resilience (e.g., Markstrom, Marshall, \& Tyron, 2000). Acceptance includes understanding emotions and thoughts as they are, without any effort to judge or to change them. This strategy is assumed to be a core facet for the treatment of a variety of disorders, and to predict different positive outcomes (e.g., Heffner, Eifert, Parker, Hernandez, \& Sperry, 2003). In contrast, suppression (i.e., suppression of negative thoughts or of showing negative feelings), avoidance (i.e., avoiding situations that might induce unpleasant feelings), and rumination (i.e., repetitively thinking about an emotion, its causes, and its consequences) have consistently been seen to be risk factors for psychopathology; for example, depressive symptoms and ill-being (Seiffge-Krenke \& Klessinger, 2000).

Aldao and colleagues (2010) examined the associations between these six ER strategies and four different psychopathologies. As expected, rumination, avoidance, and suppression showed medium to large positive correlations with an global index of all included psychopathologies (anxiety, depression, eating disorders, substance-related disorders), whereas reappraisal, problem-solving, and acceptance were negatively related to this index. These findings provide empirical support for the assumption that ER strategies can be categorized as adaptive or maladaptive over a variety of situations and contexts. It is important to note, however, that Aldao and colleagues also reported moderator effects for sample age. For instance, relationships between psychopathology and suppression were stronger for adults than for children and adolescents.

\subsection{Emotion Regulation and Psychosocial Adjustment in Early Adolescence}

The role of ER in children's psychosocial adjustment has been examined quite extensively, particularly in the early childhood period (Calkins, 2010). The acquisition of adaptive ER skills at that age has been found to have an important impact on social competence and school adjustment in later childhood (Eisenberg \& Fabes, 2006; Graziano, Reavis, Keane, \& Calkins, 2007). Moreover, numerous studies have revealed ER to be a prerequisite of both externalizing and internalizing problems. Inverse associations between adequate ER and externalizing problems (e.g., ADHD) were consistently found for toddlers, preschool, and school-aged children, as well as for adolescents, even in studies using multiple reporters and/or multiple methods of assessing ER and/or externalizing problems (cf. Eisenberg, Spinrad, \& Eggum, 2010). Frick and Morris (2004) argued that children and adolescents with reactive, emotionally driven conduct problems have difficulties in regulating their emotions and behavior when they are emotionally aroused. Therefore, they are more likely to be involved in negative socialization interactions with parents, teachers, and peers. Likewise, with regard to internalizing disorders, adequate ER strategies, such as reappraisal or acceptance, are particularly important because negative affect is often 
linked with problems, such as rumination and withdrawal. Although findings are less consistent (especially for children without externalizing comorbidities), inverse relations were found between adequate ER and internalizing problems for different ages and origins, using multiple methods (Eisenberg, Spinrad, \& Eggum, 2010).

Despite cumulative evidence on the relations between ER and psychosocial adjustment, some ambiguities and challenges remain and have been commonly addressed in ER research. Beside a general paucity of studies with older children and adolescents, Adrian, Zeman, and Veits (2011) noticed that the vast majority of published research still relies on subjective reports with single informants (either child or parent report) instead of multiple reporters. However, particularly in child psychiatry, clinicians often obtain data of multiple reporters to arrive at a child's diagnosis. On the one hand, when informants provide similar information, these patterns of findings can emphasize the accuracy of a diagnosis; on the other hand, the diagnostic process becomes much more complicated when different informants provide discrepant information. However, there is a lack of studies which systematically examine and contrast different perceptions of child and parent reports. Moreover, researchers have struggled with deciding which level of abstraction should be used when investigating ER. In contrast to global constructs on ER that only assess the valence (i.e., positive vs. negative emotions. See Laurent, Catanzaro, Joiner, Rudolph, Potter et al., 1999), the functionalist's approach has emphasized the importance of examining discrete emotions because each emotion is posited to serve a unique function with its own action tendencies (cf. Zeman, Klimes-Dougan, Cassano, \& Adrian, 2007). Consequently, research on ER can be interpreted more reasonably when focused on a specific emotion, such as anger, sadness, fear, etc. In the study presented here, we sought to address these methodological concerns by using a multi-informant design with self- and other-reports, and focusing on the regulation of anger with a questionnaire supplying distinct emotions. Anger was selected because research demonstrates dysregulated anger to be considerably important not only for externalizing, but also for internalizing problems. Moreover, deficits in anger regulation are closely related to aggression, and have been discussed as a distinctive characteristic of ADHD with and without aggression (Mullin \& Hinshaw, 2007). Likewise, aggression is a significant problem occurring most frequently in the period of adolescence, reflected in increasing incidences of (comorbid) conduct disorders from childhood to adolescence (Nock, Kazdin, Hiripi, \& Kessler, 2006).

\subsection{Emotion Regulation Profiles}

Beside our growing knowledge about the effects of adaptive and maladaptive ER strategies on psychosocial adjustment, only a few studies have been conducted to examine the interactional impact of adaptive and maladaptive ER. One reason for the lack of research concerning the interplay of adaptive and maladaptive ER strategies could be that over the last few decades, research has focused on variable-centered approaches to investigate relationships between ER and psychosocial adjustment. However, such a perspective tends to ignore that the individual is an organized whole in which each aspect of structure and process (e.g., adaptive and maladaptive ER strategies) takes on meaning based upon its role within the entire organization of the individual (Bergman \& Andersson, 2010). In contrast, advanced person-centered methods, such as latent profile analysis (LPA), include these assumptions and resolve methodological disadvantages of traditional person-centered approaches (e.g., median-split or cluster analysis), respectively. The main advantages of LPA include that a) it offers statistical tests (Bayesian information criterion [BIC]), Bootstrap Likelihood Ratio Test [BLRT]; see Nylund, Asparouhov, \& Muthén, 2007) to identify the adequate number of classes, b) existing LPA software uses model-based imputation to estimate missing data (Muthén \& Muthén, 1998-2009), and c) it provides posterior probabilities of class membership, allowing researchers to evaluate how well their model classifies individuals into the different latent classes. Moreover, LPA yields higher ecological validity as it provides the user with the really observed profiles in a sample compared to predicted values in regression models where it is unclear how many participants have really shown this particular value (Pastor et al., 2007). However, in contrast to pertinent studies in related research fields (e.g., motivational regulation; Schwinger, Steinmayr, \& Spinath, 2012), yet there are few studies which have used person-oriented approaches to study ER (for exceptions, see Hill, Degnan, Calkins, \& Keane, 2006; Zalewski, Lengua, Wilson, Trancik, \& Bazinet, 2011).

Negative yet moderate correlations have suggested that maladaptive and adaptive ER strategies should be seen as two dimensions rather than as opposite ends of one continuum. Thus, hypothetically, for adaptive and maladaptive ER strategies, it is possible to imagine adolescents who are either high in adaptive or maladaptive ER strategies, high in both dimensions, or low in both. Building on previous research, it can be assumed that 
adolescents who are high in adaptive and low in maladaptive ER strategies have the greatest benefit with respect to emotional and social competence and psychosocial adjustment. However, it is more difficult to predict the impact of the other combinations. For example, is there any compensational impact of adaptive ER strategies on maladaptive strategies, or do they appear to influence psychosocial adjustment independently? Likewise, do adolescents with high values on both adaptive and maladaptive ER benefit more than adolescents with low values on both strategies?

With respect to these questions, Stegge and Terwogt (2007) have argued that children and adolescents with internalizing problems (e.g., depression) are not only more likely to endorse maladaptive strategies, such as cognitive and behavioral avoidance and rumination, but also less likely to advocate adaptive strategies, such as active problem-solving strategies and cognitive reappraisals. Likewise, in line with the social information processing model (Crick \& Dodge, 1994), they outline that many disorders comprised in the externalizing spectrum are characterized by an anger bias that may lead to maladaptive responses to anger-eliciting situations as well as a biased appraisal of such situations. This bias may also inhibit an adequate use of adaptive anger regulation strategies, such as cognitive reappraisal or acceptance. Therefore, adolescents with a profile characterized by a lack of adaptive and a frequent use of maladaptive anger regulation strategies may be more likely to suffer from both internalizing and externalizing problems than adolescents with high values on both dimensions. In another theoretical approach, Cole, Michel, and Teti (1994) differentiated two forms of dysregulation-defined as overregulation or underregulation — of the intensity or expression of particular emotions. According to these assumptions, Mullin and Hinshaw (2007) argue that externalizing problems might particularly be linked with an underregulation of emotions, whereas internalizing problems might be more likely to be related to an overregulation of emotions. From this point of view, adolescents with high values on both adaptive and maladaptive anger regulation strategies should predominantly show higher internalizing problems, whereas adolescents who are low on both dimensions should be predominantly linked to higher externalizing problems.

\subsection{The Present Research}

The present study builds on the lack of research on ER profiles and examined the prevalence of preadolescents' anger regulation profiles and their relations with internalizing/externalizing problems and prosocial behavior in a multiple reporter study with a large sample of German 6th-grade students. Based on the theoretical rationales provided above, we proposed the following hypotheses for both parent and child reports:

Hypothesis 1. We expected different types of emotion regulators. A combination of the adaptive (high vs. low) and maladaptive (high vs. low) ER dimensions reveal four ER profiles: a) an adaptive profile (characterized by adolescents with high levels of adaptive and low levels of maladaptive strategies), b) a maladaptive profile (characterized by adolescents with high levels of maladaptive and low levels of adaptive strategies), c) a multiple profile (characterized by adolescents with high levels of both adaptive and maladaptive strategies), and d) a low profile (characterized by adolescents with low levels of both adaptive and maladaptive strategies).

Hypothesis 2. For psychosocial adjustment, not only the overall level of adaptive and maladaptive strategies, but also the relative degree of adaptive and maladaptive regulation strategies is important. Thereby, we supposed that the different profiles (see Hypothesis 1) would be divergently related to prosocial behavior and internalizing and externalizing problems. In detail, we expected that a) the adaptive profile is characterized by lower levels of internalizing and externalizing problems and higher levels of prosocial behavior than the other profiles, and that b) compared to the other profiles, the maladaptive profile is related to both higher levels of internalizing and externalizing problems. With regard to differences between the multiple and the low profile, we supposed that c) the multiple profile is predominantly associated with higher levels of internalizing problem behavior, whereas the low profile is predominantly associated with higher levels of externalizing problem behavior.

\section{Method}

\subsection{Recruitment and Procedure}

Data stem from a three-year longitudinal project supported by the German Federal Ministry for Education and Research (BMBF). Detailed information about socioeconomic status were assessed at the first measurement point (spring 2010). Data presented in the present study (ER and psychosocial adjustment) were collected at the second (spring 2011) measurement point. Our sample consists of families living in two midsized towns in northern Germany, including neighboring villages. We invited a representative selection of secondary schools to 
participate in the study. Altogether, 29 of 109 (26.6\%) schools decided to participate. Questionnaires were administered during two lesson hours to all adolescents with informed consent. Additionally, adolescents received an envelope with a parent questionnaire in which parents were asked about their parental perception of the same constructs (adolescents' ER and psychosocial adjustment). All families who completed the whole questionnaire package (adolescents' and parents' questionnaire) received a $15 €$ gift voucher.

\subsection{Participants}

Overall, $N=905$ families provided informed consent and filled out the child and the parent questionnaire at the second measurement point. In $n=608$ cases $(67 \%)$, both child and parent data were available at least at two measurement points. Only these cases were included in the analyses because we sought to control for important covariates such as socioeconomic status which had been assessed only at the first measurement point. Retention rate from the first to the second measurement point was $75 \%$. Adolescents' age at the second measurement point was between 11 and 14 years $(M=11.77, S D=.69)$ years. In all, $50.4 \%$ of adolescents were male, $76.8 \%$ attended the highest school track of secondary school (which prepares students for university studies), and 23.2\% attended the lowest school track (which prepares students for vocational training). About one third (33.7\%) of all fathers (and 22.9\% of all mothers) reported to have of a university degree, 59.4\% (69.1\%) were in possession of a training qualification, and 6.9\% (8.0\%) had no training qualification. Parents' socioeconomic status (Highest International Socio-economic Index of Occupational Status, HISEI; Ganzeboom, De Graaf, \& Treiman, 1992) had an average score of $M=54.79$ ( $S D=15.8$ ) on a scale ranging from 16 (e.g., unskilled worker) to 90 (e.g., judge), being slightly higher than the representative average in Germany ( $M=47.6$; German Federal Ministry for Education and Research, 2008). Families with lower HISEI, with children attending the lowest-track school, and in which German was spoken less frequently, showed a higher dropout, but effect sizes were rather small $(.16 \leq \phi \leq .26, p s<.05)$.

\subsection{Measures}

\subsubsection{Questionnaire for the Measurement of Emotion Regulation in Children and Adolescents (FEEL-KJ)}

The FEEL-KJ (Grob \& Smolenski, 2009) has been developed for the measurement of the habitual use of several ER strategies in response to three distinct emotions (anger, sadness, and fear). In this study, we only used the anger regulation subscale. Based on a norm sample of over 780 fourth to tenth grade students, good criterionrelated validity (e.g., significant correlations to measures of psychological well-being, depression, and expression of anger) has been demonstrated. Strategy subscales can be subsumed as two higher-order factors, representing adaptive and maladaptive ways of anger regulation. The adaptive regulation factor consists of the following strategies: Behavioral Problem-solving, Distraction, Mood-raising, Acceptance, Forgetting, Cognitive Problem-solving, and Reappraisal (14 items; sample items are displayed in the Appendix). The maladaptive anger regulation factor contains 10 items, including the following strategies: Resign, Venting, Withdrawal, Selfdefeat, and Rumination. Adolescents and parents answered the items on a 4-point Likert-type scale ( $1=$ almost never, 4 = almost always). Items were rephrased into the third person for parental assessment (e.g., "When my child is angry, (s)he starts to argue with others"). Internal consistencies were good for the adaptive ER strategies subscale ( $\alpha$ children $=.88 ; \alpha$ parent $=.87$ ) and acceptable for the maladaptive ER strategies subscale ( $\alpha$ children $=.61 ; \alpha$ parent $=.72)$.

\subsubsection{Strengths and Difficulties Questionnaire (SDQ)}

The German version of the SDQ (Goodman, 1997) is a brief behavioral screening questionnaire for children from ages 3 to 16. In a study with 930 students, Klasen et al. (2000) have shown that factor structure, reliability and validity of the German version are comparable to the English version. The questionnaire contains 25 items that ask about positive and negative psychological attributes on a 3-point Likert-type scale $(1=$ not true, $3=$ certainly true). Each item is assigned to one of the five subscales Emotional Symptoms, Conduct Problems, Hyperactivity/Inattention, Peer Relationship Problems, and Prosocial Behavior. In the case of nonclinical samples, Goodman, Lamping, and Ploubidis (2010) suggested to subsume the subscales of emotional problems and peer relationship problems, and conduct problems and inattention/hyperactivity, respectively, into two higherorder factors representing Internalizing Problems $(\alpha$ children $=.69 ; \alpha$ parent $=.75)$ and Externalizing Problems 
$(\alpha$ children $=.74 ; \alpha$ parent $=.79)$. We used these subscales as two indicators of negative psychosocial adjustment and the Prosocial Behavior subscale ( $\alpha$ children $=.68$; $\alpha$ parent $=.62$; see Appendix for sample items) to assess the degree of positive psychosocial adjustment. Internal consistencies for the present sample were comparable to validation and standardization studies (Goodman, 2001; Goodman et al., 2010; Woerner et al., 2002).

\subsection{Statistical Analyses}

A main advantage of our study refers to the multiple reporter design; that is, we collected data from both the child and parent perspective. Consequently, to strengthen the validity and generalizability of our results, we conducted all statistical procedures separately for the child and parent report data.

\subsubsection{Identification of Emotion Regulation Profiles}

In order to identify anger regulation profiles, we conducted a series of LPAs in Mplus 5.21 (Muthén \& Muthén, 1998-2009), ranging from two to six latent classes. Mean scores of adaptive and maladaptive anger regulation strategies were used as LPA indicators. Missing data were handled via model-based imputation (FIML procedure). All indicators were grand mean centered prior to analysis to facilitate interpretation. The model with the best fit was identified by comparisons of relative statistical fit measures. For this purpose, first both solutions with low Bayesian information criterion (BIC), adjusted BIC (aBIC), and Akaike information criterion (AIC) values, and those with a significant Bootstrap Likelihood Ratio Test (BLRT) were preselected. Next, the remaining solutions were compared with respect to the interpretability of their profile structure (Abar \& Loken, 2010; Marsh, Lüdtke, Trautwein, \& Morin, 2009) and classification accuracy was checked. Posterior probabilities higher than .80 and entropy near 1 indicate a statistically reliable solution (Marsh et al., 2009).

\subsubsection{Divergent Relations to Psychosocial Adjustment}

In the best fitting model, different ER profiles were compared with each other regarding prosocial behavior and internalizing and externalizing problems. These outcome variables were entered in the LPA analysis as auxiliary variables. Therefore, LPA solutions were not influenced by these variables.

\section{Results}

\subsection{LPA Solution for the Child Report}

Mean scores, standard deviations and correlations are presented in Table 1 and Table 2. With respect to child report LPA, the BIC value and the BLRT criterion indicated the best fit for the two class solution (see Table 3), whereas classification accuracy was inacceptable for this solution (.50) and clearly better for the three, four, five, and six class solutions. Therefore, the two class solution was not included in the following comparisons. Bayesian information criterion values were better for the three class solution than for the four and five class solution, but AIC values were better for the four and the five class solution. Adjusted BIC values were equal for LPA models with three, four, and five classes. For these LPA models, the BLRT criterion revealed that the four class solution had a significantly better fit than the three class solution, but no improvement of fit for the five class solution. Additionally, based on an inspection of the resulting profiles, the four class solution was considered to be more theoretically sound than the five class solution, so we decided to consider this as the final solution. Estimated means and standard errors are reported in Table 4 and anger regulation profiles are graphically presented in Figure 1. Results revealed three of the four expected combinations of adaptive and maladaptive anger regulation strategies. A large part of the adolescents (39.5\%) frequently used adaptive and infrequently used maladaptive anger regulation strategies. They were assigned to an adaptive profile (Class 1). The largest profile included $48.5 \%$ of all adolescents. We labeled this profile as the medium profile (Class 2), since it was characterized by moderate values on both maladaptive and adaptive anger regulation strategies. The third profile was labeled as maladaptive profile (Class 3), including 9.3\% of all adolescents. These adolescents frequently used maladaptive and infrequently used adaptive anger regulation strategies. Beyond that, a small subgroup (2.7\%) had a multiple profile with high values both on adaptive and maladaptive anger regulation strategies.

\subsection{Latent Profiles, Prosocial Behavior, and Problem Behavior for the Child Report}

Relations between anger regulation profiles and psychosocial adjustment were tested through mean comparisons 
Table 1. Means, standard deviations, intercorrelations, and internal consistencies (child report).

\begin{tabular}{|c|c|c|c|c|c|c|c|c|c|}
\hline & $\alpha$ & $M(S D)$ & (1) & (2) & (3) & (4) & (5) & (6) & (7) \\
\hline (1) Age & -- & $11.77(.69)$ & 1 & & & & & & \\
\hline (2) Gender & -- & $.50(.50)$ & $.11^{* *}$ & 1 & & & & & \\
\hline (3) SES & -- & $54.79(15.85)$ & $-.20^{* *}$ & .01 & 1 & & & & \\
\hline (4) Adaptive anger regulation & .88 & $2.64(.60)$ & .00 & .01 & -.04 & 1 & & & \\
\hline (5) Maladaptive anger regulation & .61 & $2.32(.47)$ & -.01 & -.02 & .01 & $-.25^{* *}$ & 1 & & \\
\hline (6) Internalizing problems & .69 & $.53(.33)$ & -.00 & $.08^{*}$ & -.07 & $-.11^{* *}$ & $.33^{* * *}$ & 1 & \\
\hline (7) Externalizing problems & .74 & $.66(.36)$ & .04 & $.18^{* *}$ & $-.09^{*}$ & $-.32^{* *}$ & $.35^{* *}$ & $.30^{* *}$ & 1 \\
\hline (8) Prosocial Behavior & .68 & $7.42(2.02)$ & $-.09^{*}$ & $-.24^{* *}$ & .05 & $.30^{* *}$ & $-.17^{* *}$ & -.05 & $-.47^{*+}$ \\
\hline
\end{tabular}

Note. ${ }^{* *} p<.01 ; \stackrel{*}{p}<.05$.

Table 2. Means, standard deviations, intercorrelations, and internal consistencies (parent report).

\begin{tabular}{cccccccccc}
\hline & $\alpha$ & $M(S D)$ & $(1)$ & $(2)$ & $(3)$ & $(4)$ & $(5)$ & $(6)$ & $(7)$ \\
\hline (1) Age & -- & $11.77(.69)$ & 1 & & & & & & \\
(2) Gender & -- & $.50(.50)$ & $.11^{* *}$ & 1 & & & & \\
(3) SES & -- & $54.79(15.85)$ & $-.19^{* *}$ & .01 & 1 & & & \\
(4) Adaptive anger regulation & .87 & $2.50(.44)$ & .02 & $-.12^{* *}$ & $-.09^{* *}$ & 1 & & & \\
(5) Maladaptive anger regulation & .72 & $2.18(.42)$ & -.04 & .02 & .05 & $-.33^{* *}$ & 1 & & \\
(6) Internalizing problems & .75 & $.27(.28)$ & .04 & .04 & $-.11^{*}$ & $-.22^{* *}$ & $.32^{* *}$ & 1 \\
(7) Externalizing problems & .79 & $.40(.30)$ & .09 & $.25^{* *}$ & $-.14^{* *}$ & $-.27^{* *}$ & $.31^{* *}$ & $.35^{* *}$ & 1 \\
(8) Prosocial Behavior & .62 & $8.34(1.57)$ & -.04 & $-.15^{* *}$ & .06 & $.24^{* *}$ & $-.23^{* *}$ & $-.25^{* *}$ & $-.33^{* *}$ \\
\hline
\end{tabular}

Note. ${ }^{* *} p<.01 ;{ }^{*} p<.05$.

Table 3. Latent profile analysis fit indices.

\begin{tabular}{|c|c|c|c|c|c|c|c|}
\hline $\mathbf{N}$ classes & $\log L$ & BIC & aBIC & AIC & BLRT & Entropy & Interpretability \\
\hline \multicolumn{8}{|l|}{ Child report } \\
\hline 2 & -1664.306 & 3373.482 & 3351.259 & 3342.611 & $p<.05$ & .507 & Difficult \\
\hline 3 & -1660.413 & 3384.928 & 3353.180 & 3340.826 & $p=.14$ & .619 & Moderate \\
\hline 4 & -1655.371 & 3394.074 & 3352.802 & 3336.741 & $p<.05$ & .609 & Good \\
\hline 5 & -1651.233 & 3405.029 & 3354.233 & 3334.467 & $p=.26$ & .700 & Moderate \\
\hline 6 & -1647.590 & 3416.973 & 3356.652 & 3333.180 & $p=.17$ & .706 & Difficult \\
\hline \multicolumn{8}{|c|}{ Parent report } \\
\hline 2 & -1674.651 & 3394.174 & 3371.950 & 3363.303 & $p<.05$ & .467 & Difficult \\
\hline 3 & -1667.888 & 3399.878 & 3368.131 & 3355.777 & $p<.05$ & .600 & Moderate \\
\hline 4 & -1663.619 & 3410.569 & 3369.297 & 3353.237 & $p=.06$ & .692 & Good \\
\hline 5 & -1659.028 & 3420.619 & 3369.823 & 3350.056 & $p<.05$ & .760 & Moderate \\
\hline 6 & -1657.221 & 3436.236 & 3375.915 & 3352.443 & $p=.42$ & .757 & Difficult \\
\hline
\end{tabular}

Note. $\log L=$ Log Likelihood; BIC = Bayesian information criterion; aBIC = adjusted Bayesian information criterion; AIC = Akaike information criterion; BLRT = Bootstrap Likelihood Ratio Test. Minimal BIC indicates best relative fit. Significant BLRT denotes an improvement of fit given the additional class (e.g., three class model fits significantly better than the two class model). Bold values represent the best fitting model. 
Table 4. Profile means and standard errors for anger regulation strategies and adjustment.

\begin{tabular}{ccccc}
\hline Child report & $\mathbf{1}$ (adaptive) & $\mathbf{2}$ (medium) & $\mathbf{3}$ (maladaptive) & $\mathbf{4}$ (multiple) \\
\hline Adaptive anger regulation & $.79(.08)$ & $-.26(.15)$ & $-1.50(.22)$ & $1.03(.17)$ \\
Maladaptive anger regulation & $-.52(.12)$ & $.14(.11)$ & $.68(.18)$ & $1.37(.25)$ \\
Internalizing problems & $-.18^{\mathrm{a}}(.07)$ & $.03^{\mathrm{b}}(.06)$ & $.31^{\mathrm{c}}(.14)$ & $.51^{\mathrm{bc}}(.29)$ \\
Externalizing problems & $-.35^{\mathrm{a}}(.06)$ & $.09^{\mathrm{b}}(.06)$ & $.68^{\mathrm{c}}(.15)$ & $.32^{\mathrm{bc}}(.27)$ \\
Prosocial behavior & $.29^{\mathrm{a}}(.06)$ & $-.06^{\mathrm{b}}(.06)$ & $-.47^{\mathrm{c}}(.15)$ & $-.12^{\mathrm{abc}}(.27)$ \\
\hline Parent report & $\mathbf{1}$ (adaptive) & $\mathbf{2}$ (medium) & $\mathbf{3}$ (maladaptive) & $\mathbf{4}$ (multiple) \\
\hline Adaptive anger regulation & $.60(.11)$ & $-.43(.21)$ & $-2.08(.41)$ & $1.63(.90)$ \\
Maladaptive anger regulation & $-.65(.19)$ & $.42(.09)$ & $1.16(.24)$ & $1.38(.74)$ \\
Internalizing problems & $-.23^{\mathrm{a}}(.05)$ & $.13^{\mathrm{b}}(.06)$ & $1.05^{\mathrm{c}}(.39)$ & $.51^{\mathrm{abc}}(.50)$ \\
Externalizing problems & $-.27^{\mathrm{a}}(.05)$ & $.18^{\mathrm{b}}(.06)$ & $.86^{\mathrm{c}}(.29)$ & $.64^{\mathrm{bc}}(.50)$ \\
Prosocial behavior & $.27^{\mathrm{a}}(.06)$ & $-.12^{\mathrm{b}}(.06)$ & $-.45^{\mathrm{b}}(.25)$ & $.30^{\mathrm{ab}}(.40)$ \\
\hline
\end{tabular}

Note. Different subscripts indicate significant differences between groups $(p<.05)$.
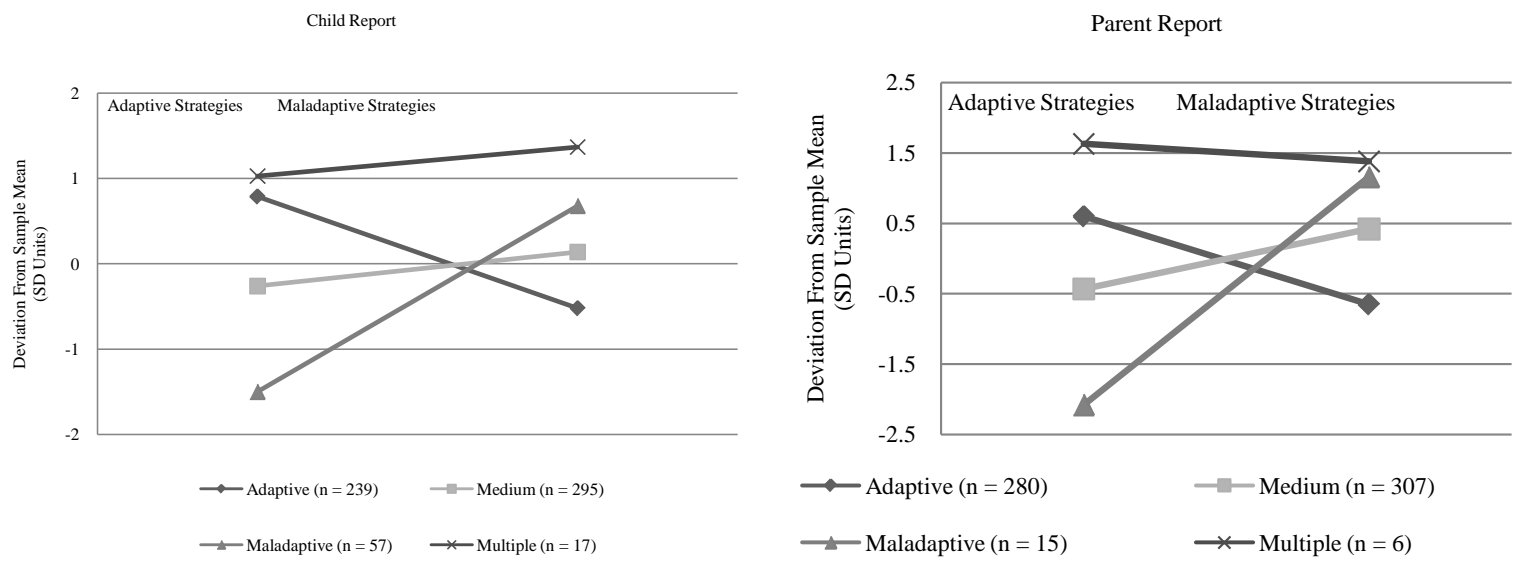

Figure 1. Profile means in adaptive and maladaptive anger regulation for child and parent report four class solution.

between profiles which were additionally provided by latent profile analyses in Mplus. In line with our hypotheses, the adaptive profile had significantly lower values on internalizing and externalizing problems compared to all other profiles and significantly higher values on prosocial behavior compared to all but the multiple profile ( $p$ s $<.05$; see Table 4). Moreover, the maladaptive and the multiple profiles were characterized by significantly higher values on internalizing (maladaptive $\chi^{2}=9.43$; multiple $\chi^{2}=5.31$, $p$ s $<.05$ ) and externalizing problems (maladaptive $\chi^{2}=36.58$; multiple $\chi^{2}=5.70$, $p$ s $<.05$ ) compared to the adaptive profile. Since LPA had not revealed an additional low profile, our hypothesis on divergent relations of the multiple and the low profile with problem behavior could not be tested. Instead, we examined divergent relations to internalizing and externalizing problems between the maladaptive and the multiple profiles; however, effects did not reach statistical significance due to high standard deviations in these small profiles. Nevertheless, by tendency, students holding the maladaptive profile showed higher values on externalizing problems compared to the multiple profile $(M=.68$, $S D=.15$ vs. $M=.32, S D=.27$ ), whereas students holding the multiple profile showed higher values on internalizing problems compared to the maladaptive profile $(M=.51, S D=.29$ vs. $M=.31, S D=.14)$.

\subsection{LPA Solution for Parent Report}

In order to interpret the parent report LPA results, we used the same procedure as for the child report. BLRT 
values indicated that the five class solution had the best fit. BIC values were best for the three and the four class solution, but AIC values were better for the five class solution. Adjusted BIC values were equal for LPA models with three, four, and five classes. The four, five, and six class solutions provided acceptable classification accuracy, indicated by entropies near .80, particularly for the five and six class solution. However, interpretability was difficult for the five class solution due to two very small profiles, including only two adolescents each. Indeed, interpretability was good for the four class solution, so we decided to consider this as the best solution (Table 3). Altogether, the parent report profiles were very similar to the child report profiles (see Figure 1). More than $90 \%$ of the adolescents were classified into an adaptive profile (Class $1 ; 46.1 \%$ ) or a medium profile (Class 2; 50.5\%). According to the child report data, the remaining adolescents were assigned to two classes labeled as maladaptive profile (Class 3; 2.4\%) and multiple profile (Class 4; 1.0\%).

\subsection{Latent Profiles, Prosocial Behavior, and Problem Behavior for the Parent Report}

Overall, results from comparisons of parent report profiles were similar to the child report results. In accordance with the child report results, the adaptive profile was associated with significantly lower values on internalizing and externalizing problems compared to the medium $\left(\chi^{2}=14.40 ; \chi^{2}=23.82 ; p s<.01\right)$ and the maladaptive profiles $\left(\chi^{2}=10.26 ; \chi^{2}=14.68 ; p s<.01\right.$; see Table 4$)$. Again, the multiple profile was characterized by apparently higher values on both internalizing and externalizing problems, but differences between the adaptive and multiple profiles did not reach statistical significance in most comparisons, probably due to high standard deviations. Moreover, just as in the child report findings, the maladaptive-but not the multiple profile-had significantly lower values on prosocial behavior compared to the adaptive profile $\left(\chi^{2}=7.81, p<.01\right)$. Similar to the child report results, our hypothesis regarding differences between the low and the multiple profile could not be tested since LPA have not revealed a low profile. Moreover, no significant divergent relations to internalizing and externalizing problems were found between the maladaptive and the multiple profiles. However, in contrast to the child report findings, an inspection of mean values indicated that students holding the maladaptive profile showed slightly higher values not only on externalizing problems $(M=.86, S D=.29$ vs. $M=.64, S D=.50)$, but also on internalizing problems $(M=1.05, S D=.39$ vs. $M=.51, S D=.50)$ compared to the multiple profile.

\section{Discussion}

The goals of the present study were to examine anger regulation profiles and their concurrent relations to psychosocial adjustment in early adolescence. Latent profile analysis solutions revealed four profiles which were identical across child and parent report data and which were characterized by different combinations of adaptive and maladaptive strategies, respectively. As expected, the child and the parent report revealed an adaptive, a maladaptive, and a multiple profile, supporting a two-dimensional approach of adaptive and maladaptive ER. In contrast to our expectations, the remaining profile was structured differently, reflecting a medium profile rather than a low profile. The absence of a low profile is not in line with our theoretical assumptions and findings from previous research (e.g., Mullin \& Hinshaw, 2007). We think, however, that one reason of this finding may have to do with social desirability which may have led to a general tendency to response positively to at least some of the items on anger regulation strategies. Moreover, adolescents who are rather unregulated may also have a biased self-perception when they report on their anger regulation capacities and therefore may tend to overestimate the frequency of using several anger regulation strategies. Parents, on the other hand, may have some difficulties to report on their children's anger regulation abilities when their child is rather unregulated, and may overestimate the use of anger regulation strategies particularly with respect to internal strategies. Altogether, the medium and the multiple profile groups identified in the child and the parent report data indicate that adaptive and maladaptive anger regulation strategies represent two dimensions that can be adjusted independently within the individual child.

\subsection{Divergent Relations between Anger Regulation Profiles and Adjustment}

Overall, comparisons of anger regulation profiles supported our expectations on divergent relations between different anger regulation profiles and psychosocial adjustment (Hypothesis 2). The child and the parent report maladaptive profiles — but not the multiple profiles - were characterized by significant lower prosocial behavior compared to the adaptive profiles. With respect to internalizing and externalizing problems, there were several 
differences between the parent and the child report data. Child reported internalizing problems were highest in the multiple profile, whereas externalizing problems were highest in the maladaptive profile. For the parent report profiles, in contrast, we found both internalizing and externalizing problems to be highest in the maladaptive profile. The child report findings are in line with our expectations based on Mullin and Hinshaw (2007) who argued that externalizing problems might be linked with an underregulation of emotions, whereas internalizing problems might be more likely to be related to an overregulation of emotions. Accordingly, it seems to be reasonable to regard the interaction of maladaptive and adaptive anger regulation strategies when assessing the potential risk of internalizing and externalizing problems. In contrast, parent report results provide some weak support for the assumption that adaptive anger regulation strategies may have a compensational effect in adolescents with high levels of maladaptive anger regulation strategies.

Discrepancies between child and parent report findings have been observed for many years (Achenbach, McConaughy, \& Howell, 1987; De Los Reyes \& Kazdin, 2005). One possible explanation refers to the parents’ perception of their own child. Increased maladaptive strategies combined with a lack of prosocial behavior might especially make a significant difference on parental perception of psychosocial adjustment because these adolescents attract more negative attention than the multiple profile adolescents who do not show decreased prosocial behavior. On the other hand, it is also possible that parents who consider their adolescents to show strong problem behavior (e.g., aggressive behavior) are particularly sensitized to related maladaptive ER strategies (e.g., venting) and do not perceive whether their children also use adaptive strategies. Future studies using multiple reporters are needed to investigate possible reasons of such discrepancies more explicitly. However, recent studies emphasize that researchers can extract meaningful information from discrepancies between child and parent reports (e.g., Hourigan, Goodman, \& Southam-Gerow, 2011). In this regard, our findings indicate that psychopathologies seem less likely to be perceived by parents of adolescents with a multiple anger regulation profile. Thus, specific attention should be paid not only to adolescents with maladaptive anger regulation profiles, but also to adolescents with multiple anger regulation profiles in diagnostics and prevention settings. However, despite considerable mean differences between the multiple and the maladaptive profiles, findings should be interpreted with care because these differences did not reach statistical significance due to high standard deviations in the extreme profiles.

\subsection{Implications}

Our findings have several implications for clinical child and adolescent psychology. First, maladaptive and adaptive anger regulation seem to be two different dimensions rather than two opposite ends of one continuum. Therefore, several dysfunctional combinations may emerge in adolescents with anger regulation difficulties. Identifying the individual interplay of adaptive and maladaptive anger regulation strategies may yield important information about the risk of internalizing or externalizing problems. Moreover, it appears promising to address both adaptive and maladaptive anger regulation strategies in prevention and/or intervention programs, since findings support the assumption that training of adaptive anger regulation strategies may not necessarily replace maladaptive ones. Finally, child report findings provide support that emotion regulation intervention programs may be adjusted according to specific forms of problem behavior. Particularly, it might be promising to integrate additional training of adaptive anger regulation strategies in case of externalizing problem behavior. Initial ER trainings have been developed for children (e.g., Wyman, Cross, Brown, Yu, Tu, \& Eberly, 2010) and adolescents (e.g., Schuppert, Giesen-Bloo, van Gemert, Wiersema, Minderaa et al., 2009). Our findings support the relevance of such ER training approaches for clinical child and adolescent psychology.

\subsection{Strengths and Limitations}

Overall, our study has important strengths. First, we used LPA as a sophisticated method to examine the interplay of adaptive and maladaptive ER strategies. Second, we focused on the understudied period of early adolescence. A final strength is the multiple-reporter design. However, the study also has several limitations. More research is needed to examine if the profiles identified in the present study (e.g., the medium profile) can be replicated in future studies. Despite consistent findings in the child and parent report data and despite the large sample size, findings should be interpreted with care due to the fact that only a minority of adolescents (3.4\% to $12 \%)$ was classified into the extreme profiles. Moreover, findings may be limited to community samples. It is unclear whether similar profiles and relations with psychosocial adjustment would emerge in clinical samples. 
Another limitation refers to the cross-sectional design of the study. Further longitudinal studies are needed to examine causal relations between anger regulation profiles and adjustment. Finally, the findings from the present study can neither be generalized to other emotions (e.g., sadness, anxiety, or joy) nor do they allow for comparing different emotions.

\section{Conclusion}

- The present study revealed four different combinations of frequent vs. infrequent use of adaptive vs. maladaptive anger regulation strategies in a large sample of early adolescents, including an adaptive, a maladaptive, a multiple and a medium — but unexpectedly no low—anger regulation profile.

- Findings suggest that maladaptive and adaptive anger regulation strategies seem to be two different dimensions rather than two opposite ends of one continuum.

- It may be reasonable to regard the interaction of maladaptive and adaptive anger regulation strategies when assessing the potential risk of internalizing and externalizing problems. A maladaptive profile seems to be associated both with internalizing and externalizing problems, whereas a multiple profile seems to be particularly linked to internalizing problems.

- It appears promising to address both adaptive and maladaptive anger regulation strategies in prevention and/ or intervention programs, since findings support the assumption that training of adaptive anger regulation strategies may not necessarily replace maladaptive ones.

\section{Acknowledgements}

The studies reported in this article were supported by a grant from the German Federal Ministry for Education and Research (BMBF; 01GJ0984) to the third author. We wish to thank Anastasia Byler for native-speaker advice.

\section{References}

Abar, B., \& Loken, E. (2010). Self-Regulated Learning and Self-Directed Study in a Precollege Sample. Learning and Individual Differences, 20, 25-29. http://dx.doi.org/10.1016/j.lindif.2009.09.002

Achenbach, T. M., McConaughy, S. H., \& Howell, C. T. (1987). Child/Adolescent Behavioral and Emotional Problems: Implications of Cross-Informant Correlations for Situational Specifity. Psychological Bulletin, 101, 213-232. http://dx.doi.org/10.1037/0033-2909.101.2.213

Adrian, M., Zeman, J., \& Veits, G. (2011). Methodological Implications of the Affect Revolution: A 35-Year Review of Emotion Regulation Assessment in Children. Journal of Experimental Child Psychology, 110, 171-197. http://dx.doi.org/10.1016/j.jecp.2011.03.009

Aldao, A., Nolen-Hoeksema, S., \& Schweizer, S. (2010). Emotion-Regulation Strategies across Psychopathology: A MetaAnalytic Review. Clinical Psychology Review, 30, 217-237. http://dx.doi.org/10.1027/0044-3409/a000025

Bergman, L. R., \& Andersson, H. (2010). The Person and the Variable in Developmental Psychology. The Journal of Psychology, 218, 155-165. http://dx.doi.org/10.1027/0044-3409/a000025

Calkins, S. D. (2010). Commentary: Conceptual and Methodological Challenges to the Study of Emotion Regulation and Psychopathology. Journal of Psychopathology and Behavioral Assessment, 32, 92-95. http://dx.doi.org/10.1007/s10862-009-9169-6

Cole, P. M., Michel, M. K., \& Teti, L. O. D. (1994). The Development of Emotion Regulation and Dysregulation: A Clinical Perspective. Monographs of the Society for Research in Child Development, 59, 73-102. http://dx.doi.org/10.2307/1166139

Crick, N. R., \& Dodge, K. A. (1994). A Review and Reformulation of Social Information-Processing Mechanisms in Children's Social Adjustment. Psychological Bulletin, 115, 74. http://dx.doi.org/10.1037/0033-2909.115.1.74

De Los Reyes, A., \& Kazdin, A. E. (2005). Informant Discrepancies in the Assessment of Childhood Psychopathology: A Critical Review, Theoretical Framework, and Recommendations for Further Study. Psychological Bulletin, 131, $483-509$. http://dx.doi.org/10.1037/0033-2909.131.4.483

Eisenberg, N., \& Fabes, R. (2006). Emotion Regulation and Children’s Socioemotional Competence. In L. Balter, \& C. Tamis-LeMonda (Eds.), Child Psychology: A Handbook of Contemporary Issues (2nd ed., pp. 357-381). New York: Psychology Press.

Eisenberg, N., Spinrad, T. L., \& Eggum, N. D. (2010). Emotion-Related Self-Regulation and Its Relation to Children’s Ma- 
ladjustment. Annual Review of Clinical Psychology, 6, 495-525. http://dx.doi.org/10.1146/annurev.clinpsy.121208.131208

Frick, P. J., \& Morris, A. S. (2004). Temperament and Developmental Pathways to Conduct Problems. Journal of Clinical Child and Adolescent Psychology, 33, 54-68. http://dx.doi.org/10.1207/S15374424JCCP3301_6

Ganzeboom, H. B. G., De Graaf, P. M., \& Treiman, D. J. (1992). A Standard International Socio-Economic Index of Occupational Status. Social Science Research, 21, 1-56. http://dx.doi.org/10.1016/0049-089X(92)90017-B

German Federal Ministry of Education and Research (2008). Berufsbildungsbericht. Bonn.

Goodman, R. (1997). The Strengths and Difficulties Questionnaire: A Research Note. Journal of Child Psychology and Psychiatry, 38, 581-586. http://dx.doi.org/10.1111/j.1469-7610.1997.tb01545.x

Goodman, R. (2001). Psychometric Properties of the Strengths and Difficulties Questionnaire. Journal of the American Academy of Child and Adolescent Psychiatry, 40, 1337-1345. http://dx.doi.org/10.1097/00004583-200111000-00015

Goodman, A., Lamping, D. L., \& Ploubidis, G. B. (2010). When to Use Broader Internalizing and Externalizing Subscales Instead of the Hypothesized Five Subscales on the Strengths and Difficulties Questionnaire (SDQ): Data from British Parents, Teachers and Children. Journal of Abnormal Child Psychology, 38, 1179-1191. http://dx.doi.org/10.1007/s10802-010-9434-x

Graziano, P., Reavis, R., Keane, S., \& Calkins, S. (2007). The Role of Emotion Regulation in Children’s Early Academic Success. Journal of School Psychology, 45, 3-19. http://dx.doi.org/10.1016/j.jsp.2006.09.002

Grob, A., \& Smolenski, C. (2009). Fragebogen zur Erhebung der ER bei Kindern und Jugendlichen (FEEL-KJ). Questionnaire for the Measurement of Emotion Regulation in Children and Adolescents. Bern: Huber.

Heffner, M., Eifert, G. H., Parker, B. T., Hernandez, D. H., \& Sperry, J. A. (2003). Valued Directions: Acceptance and Commitment Therapy in the Treatment of Alcohol Dependence. Cognitive and Behavioral Practice, 10, 378-383. http://dx.doi.org/10.1016/S1077-7229(03)80055-X

Hill, A. L., Degnan, K. A., Calkins, S. D., \& Keane, S. P. (2006). Profiles of Externalizing Behavior Problems for Boys and Girls across Preschool: The Roles of Emotion Regulation and Inattention. Developmental Psychology, 42, 913-928. http://dx.doi.org/10.1037/0012-1649.42.5.913

Hourigan, S. E., Goodman, K. L., \& Southam-Gerow, M. A. (2011). Discrepancies in Parents' and Children’s Reports of Child Emotion Regulation. Journal of Experimental Child Psychology, 110, 198-212. http://dx.doi.org/10.1016/j.jecp.2011.03.002

Klasen, H., Woerner, W., Wolke, D., Meyer, R., Overmeyer, S., Kaschnitz, W., Rothenberger, A., \& Goodman, R. (2000). Comparing the German Versions of the Strengths and Difficulties Questionnaire (SDQ-Deu) and the Child Behavior Checklist. European Child and Adolescent Psychiatry, 9, 271-276. http://dx.doi.org/10.1007/s007870070030

Laurent, J., Catanzaro, S., Joiner, T., Rudolph, K., Potter, K., Lambert, S., Osborne, L., \& Gathright, T. (1999). A Measure of Positive and Negative Affect for Children: Scale Development and Preliminary Validation. Psychological Assessment, 11, 326-338. http://dx.doi.org/10.1037/1040-3590.11.3.326

Markstrom, C. A., Marshall, S. K., \& Tryon, R. J. (2000). Resiliency, Social Support, and Coping in Rural Low-Income Appalachian Adolescents from Two Racial Groups. Journal of Adolescence, 23, 693-703. http://dx.doi.org/10.1006/jado.2000.0353

Marsh, H. W., Lüdtke, O., Trautwein, U., \& Morin, A. J. S. (2009). Classical Latent Profile Analysis of Academic SelfConcept Dimensions: Synergy of Person- and Variable-Centered Approaches to Theoretical Models of Self-Concept. Structural Equation Modeling: A Multidisciplinary Journal, 16, 191-225. http://dx.doi.org/10.1080/10705510902751010

Morris, A. S., Silk, J. S., Steinberg, L., Myers, S., \& Robinson, L. R. (2007). The Role of the Family Context in the Development of Emotion Regulation. Social Development, 16, 361-388. http://dx.doi.org/10.1111/j.1467-9507.2007.00389.x

Mullin, B. C., \& Hinshaw, S. P. (2007). Emotion Regulation and Externalizing Disorders in Children and Adolescents. In J. J. Gross (Ed.), Handbook of Emotion Regulation (pp. 523-541). New York: Guilford Press.

Muthén, L. K., \& Muthén, B. O. (1998-2009). Mplus User’s Guide (5th ed.). Los Angeles, CA: Muthén \& Muthén.

Nock, M., Kazdin, A., Hiripi, E., \& Kessler, R. (2006). Prevalence, Subtypes, and Correlates of DSM-IV Conduct Disorder in the National Comorbidity Survey Replication. Psychological Medicine, 36, 699-710. http://dx.doi.org/10.1017/S0033291706007082

Nylund, K. L., Asparouhov, T., \& Muthén, B. (2007). Deciding on the Number of Classes in Latent Class Analysis and Growth Mixture Modeling: A Monte Carlo Simulation Study. Structural Equation Modeling: A Multidisciplinary Journal, 14, 535-569. http://dx.doi.org/10.1080/10705510701575396

Schuppert, H. M., Giesen-Bloo, J., van Gemert, T. G., Wiersema, H. M., Minderaa, R. B., Emmelkamp, P. M., \& Nauta, M. H. (2009). Effectiveness of an Emotion Regulation Group Training for Adolescents-A Randomized Controlled Pilot Study. Clinical Psychology and Psychotherapy, 16, 467-478. http://dx.doi.org/10.1002/cpp.637

Schwinger, M., Steinmayr, R., \& Spinath, B. (2012). Not All Roads Lead to Rome-Comparing Different Types of Motiva- 
tional Regulation Profiles. Learning and Individual Differences, 22, 269-279. http://dx.doi.org/10.1016/j.lindif.2011.12.006

Seiffge-Krenke, I., \& Klessinger, N. (2000). Long-Term Effects of Avoidant Coping on Adolescents’ Depressive Symptoms. Journal of Youth and Adolescence, 29, 617-630. http://dx.doi.org/10.1023/A:1026440304695

Stegge, H., \& Terwogt, M. M. (2007). Awareness and Regulation of Emotion in Typical and Atypical Development. In J. J. Gross (Ed.), Handbook of Emotion Regulation (pp. 269-286). New York: Guilford Press.

Southam-Gerow, M. A., \& Kendall, P. C. (2002). Emotion Regulation and Understanding. Implications for Child Psychology and Therapy. Clinical Psychology Review, 22, 189-222. http://dx.doi.org/10.1016/S0272-7358(01)00087-3

Thompson, R. A. (1994). Emotion Regulation: A Theme in Search of a Definition. In N. A. Fox (Ed.), Monographs of the Society for Research in Child Development (Vol. 59, pp. 25-52). Chicago.

Woerner, W., Becker, A., Friedrich, C., Rothenberger, A., Klasen, H., \& Goodman, R. (2002). Normierung und Evaluation der deutschen Elternversion des Strengths and Difficulties Questionnaire (SDQ): Ergebnisse einer repräsentativen Felderhebung. Zeitschrift für Kinder- und Jugendpsychiatrie und Psychotherapie, 30, 105-112.

Wyman, P. A., Cross, W., Brown, C. H., Yu, Q., Tu, X., \& Eberly, S. (2010). Intervention to Strengthen Emotional SelfRegulation in Children with Emerging Mental Health Problems: Proximal Impact on School Behavior. Journal of Abnormal Child Psychology, 38, 707-720. http://dx.doi.org/10.1007/s10802-010-9398-x

Zeman, J., Klimes-Dougan, B., Cassano, M., \& Adrian, M. (2007). Measurement Issues in Emotion Research with Children and Adolescents. Clinical Psychology: Science and Practice, 14, 377-401. http://dx.doi.org/10.1111/j.1468-2850.2007.00098.x 